\title{
The influence of torsion on braided rope performance, modelling and tests
}

\author{
Davies Peter ${ }^{1,{ }^{*}}$, Durville Damien ${ }^{2}$, Vu Thanh Do ${ }^{1,2}$ \\ ${ }^{1}$ IFREMER Centre de Bretagne, Marine Structures laboratory, Plouzané 29280, France \\ ${ }^{2}$ LMSSMat, CNRS UMR8579, Centrale Supélec, Université Paris-Saclay, France \\ *Corresponding author : Peter Davies, email address : peter.davies@ifremer.fr
}

\begin{abstract}
:
Twist may be introduced accidentally into braided ropes during operations at sea, and it is important to know how this will affect both rope integrity and safety coefficients. This paper describes the use of simulation tools to evaluate how twisting can change the tensile properties of braided ropes. The case of a $300 \mathrm{kN}$ break load 12 strand braided HMPE rope is examined. An original numerical modelling approach is presented, and results are compared with results from tensile tests performed on ropes with different levels of twist. A drop in strength of around $4 \%$ per turn per meter, and an increase in elongation, were observed as the number of turns per meter increased, corresponding to progressive removal of the load-bearing capacity of half the braided strands. The model shows how load is progressively redistributed within the braid. However, very high twist levels $(>10 \mathrm{~T} / \mathrm{m})$ are required to reduce strength below $50 \%$ of the initial value.
\end{abstract}

\section{Highlights}

- New experimental data are presented from tests on 12 strand braided HMPE ropes. A new finite element model has been developed to simulate the effect of twist on both braid geometry and mechanical behavior under tensile loading. This model provides a reasonable prediction of loadstrain plots for a range of twist levels up to 5 turns $/ \mathrm{m}$. Analysis of these results and published data has provided values from over 40 tests on 8 - and 12- strand braided ropes, which indicate a mean loss in residual strength of around $5 \%$ per turn per meter.

Keywords : Torsion, Rope, Braid, Numerical model 


\section{Introduction}

Braided fiber ropes are being used for deep sea handling applications, where their low weight results in more efficient lifting compared to steel wires [1,2]. Other safety-critical applications where braided ropes have replaced steel include mining ropes, cranes and towing lines [3]. There have been various studies of torsion in steel wire ropes, e.g. [4-6], but very few for synthetic fibre ropes. However, the low torsional stiffness of fibre ropes offers little resistance to rotation about the rope axis, or twist, compared to steel, and this may modify the rope performance and limit applications. Braided ropes are often selected as they are torque balanced, so pure tension should not induce rotation (unlike some other less 
balanced twisted constructions), but asymmetry in the payload and irregular loads due to currents for example, may still induce rotation. Also, adding a synthetic rope section to extend the depth capabilities of steel mooring lines may result in serious coupling problems [7]. For marine operations involving costly vessels and expensive payloads it is therefore important to understand how this twist will affect the rope and to quantify its effect on safety coefficients.

There has been some previous work to study this loading mechanism. For example, Davies \& O'Hear studied 8-strand braided ropes of 365 to $650 \mathrm{kN}$ break loads and measured a mean drop in break strength of $6.8 \%$ per turn/meter of twist [8]. A total of 20 tests was performed in that study. In another investigation, aiming to understand the origins of strength losses in HMPE tug-lines after service [9], a number of 12 strand and $8 \times 3$ strand braids up to $32 \mathrm{~mm}$ diameter ( $835 \mathrm{kN}$ break load) were tested. A drop of 4-6\% per turn/meter was measured.

In one of those previous studies [8] both a linear geometrical approach and a commercial software code known as FRM (Fibre Rope Modeller ${ }^{\mathrm{TM}}$ ), developed by TTI [10-12], have been used to model twist effects on tensile load-strain behaviour. The latter is a hierarchical analysis which calculates rope behaviour using the virtual work principle, taking the nonlinear yarn characteristics and the geometry at each level to predict the response of ropes of any size. These two models both provided predictions which tend to overestimate the influence of twist on break strength.

In the present work a new set of tests has been performed on ten 12-strand braided HMPE ropes, and experimental results will be presented first. A finite element model has then been applied, Multifil. This model uses an implicit solver within a quasi-static framework. The initial configuration of a braided rope is determined first as a mechanical equilibrium, starting from an arbitrary configuration showing large inter-penetrations between yarns, and letting contact-friction interactions gradually move yarns away from each other, until fulfilling the selected weaving pattern. A similar approach was developed previously for woven fabrics [13-15]. It was adapted to the case of braided structures by Vu et al.[16]. The finite element approach employed, whose theoretical background is described in [17], solves the mechanical equilibrium of general beam assemblies subjected to large deformations, and developing contact-friction interactions. Yarns are modeled by means of finite strain 
beam elements, contact-friction interactions between yarns are detected and modelled. This software has been described in detail in a previous paper [16], so only the specific model, input data and conditions used to describe the influence of torsion will be presented here.

\section{Materials and Methods}

The rope samples were 12 strand AmSteelBlue ${ }^{\text {TM }}$ braids supplied by Samson Ropes; these are composed of SK75 Dyneema ${ }^{\mathrm{TM}}$ fibres. Nominal rope diameter was $18 \mathrm{~mm}$ and minimum break load was $236 \mathrm{kN}$. The braid repeat length was $120 \mathrm{~mm}$.

Tensile testing was performed initially on the assembled yarns which make up the rope, in order to establish input values (force-strain data) for modeling. This has been described previously [16], so only the rope tests will be described here. The rope samples were 8 meters long, spliced at each end over a 1.5 meter length with loops, and tested on a $1000 \mathrm{kN}$ capacity, 10 meter long test frame, Figure 1.

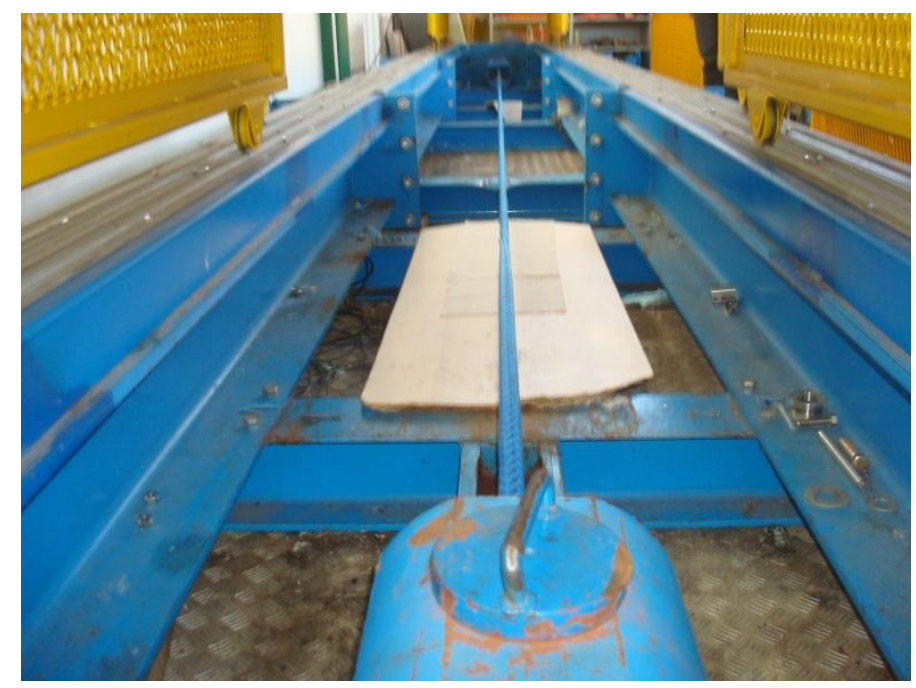

Figure 1. Braided rope on tensile test frame before test.

The procedure was to attach the ends of the rope to the machine by placing the eye spliced loops over $100 \mathrm{~mm}$ diameter steel pin. Each rope sample was then subjected to 5 loadunload bedding-in cycles between 5 and $100 \mathrm{kN}$ and was then unloaded. The required twist was introduced by rotating removing one pin and twisting manually. The free end was then replaced over the steel pin. The number of turns per meter was defined by dividing the 
number of turns by the measured free length (the distance between the ends of the splices, typically about 5.5 meters). It was noted that the spliced sections did not rotate due to their higher stiffness. before ramping to failure at a constant load rate of $100 \mathrm{kN} /$ minute. Load and piston displacement were measured continuously, together with the movements of two markers in the central section of the rope close to the ends of the splices. These movements were recorded by digital cameras fixed to a gantry above the test frame. The initial distance between the markers at a reference load of $5 \mathrm{kN}$ defined the gauge length $\mathrm{L}_{0}$; the central rope section strain was defined as the difference between the axial displacements of the two markers throughout the test, recorded at a frequency of $1 \mathrm{~Hz}$, divided by the gauge length $\mathrm{L}_{\mathrm{o}}$. In-house software using image analysis tools enabled the force-strain plots to be calculated. Ten rope samples were tested.

\section{Test results}

Figure 2 shows examples of the rope under different levels of twist. It should be emphasized that the highest twist levels are quite extreme, and very unlikely to be encountered in practice.

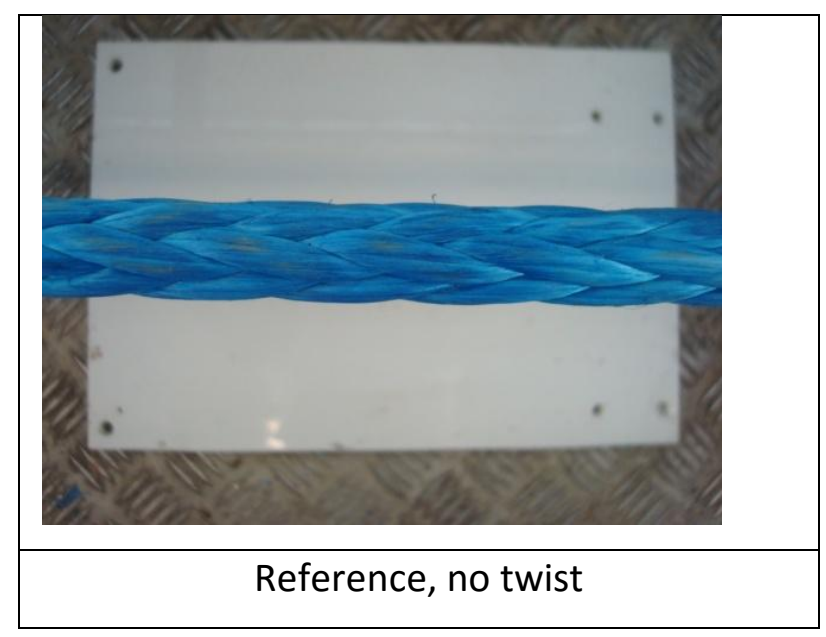




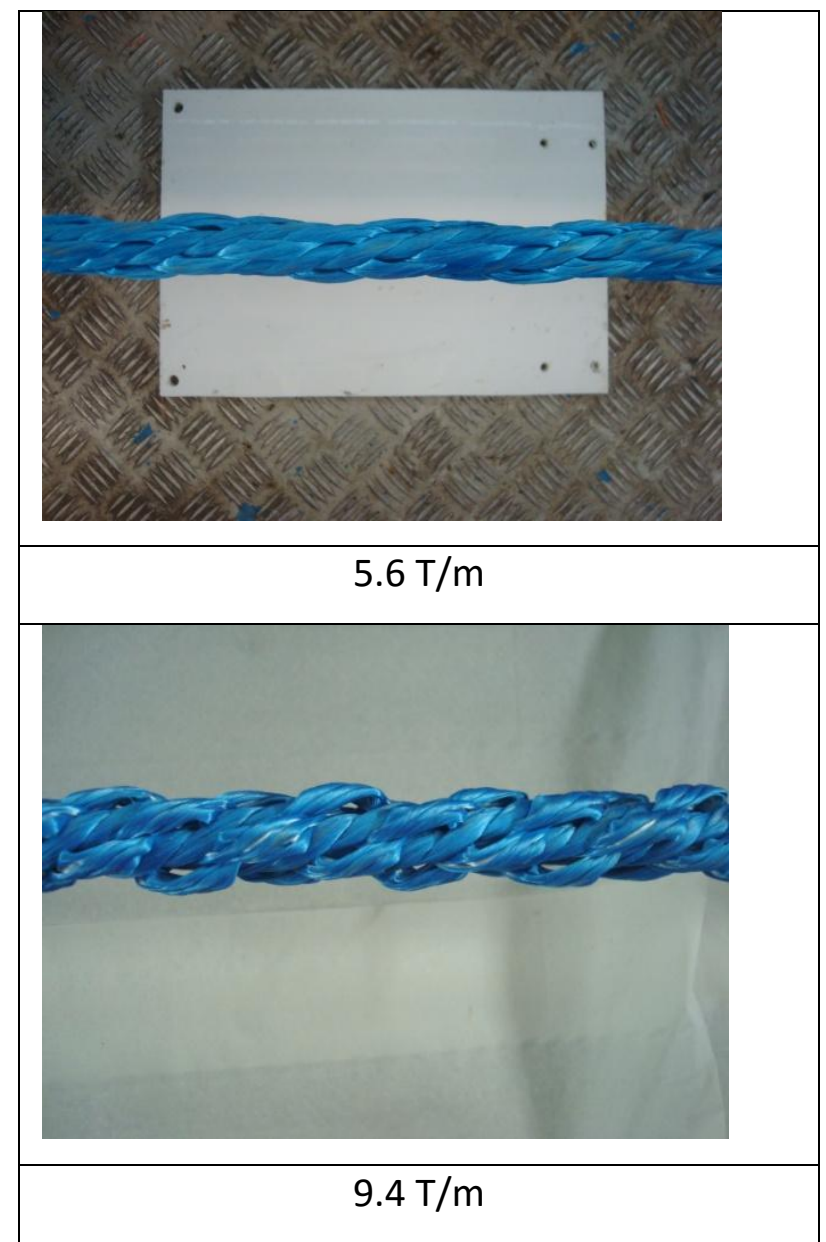

Figure 2. Examples of twisted rope samples before testing to failure

Figure 3 shows examples of the load-strain plots for the bedding-in tests before twisting.

Plots are shown for four samples. These samples were all taken from the same reel of rope and should be identical, so the small differences in response are due to variability between the samples and the nominally identical test conditions. 


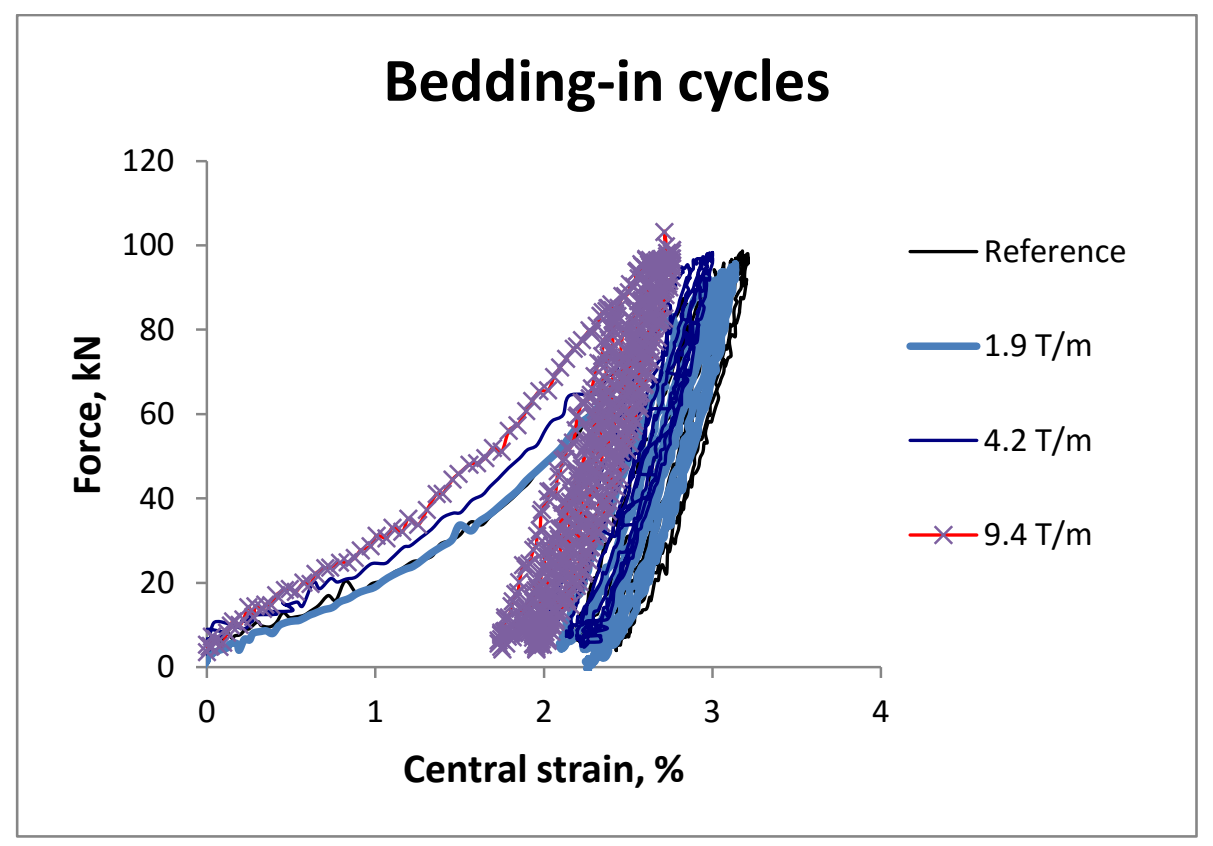

Figure 3. Examples of bedding-in cycles before twisting.

Overall the response to bedding-in was very similar for all samples, with a final strain after unloading between 2.0 and $2.7 \%$. Table 1 summarizes the residual strains after bedding-in for all samples, before the ramp to failure.

\begin{tabular}{|ccccc|}
\hline $\begin{array}{c}\text { Twist, } \\
\text { t/m }\end{array}$ & Strain at end of & Strain to failure, & Break load, & Stiffness, \\
beference & 2.4 & $\%$ & $\mathrm{kN}$ & $\mathrm{kN} / \%$ \\
1.9 & 2.3 & 2.8 & 298 & 111.6 \\
3.1 & 2.4 & 3.4 & 271 & 71.3 \\
4.2 & 2.3 & 3.8 & 261 & 58.1 \\
5 & 2.5 & 4.1 & 244 & 50.5 \\
5.6 & 2.5 & 4.8 & 218 & 43.1 \\
6.5 & 2.5 & 5.1 & 212 & 40.1 \\
7.4 & 2.5 & 5.6 & 204 & 36.8 \\
9.4 & 2.0 & 6.6 & 206 & 32.4 \\
11.2 & 2.7 & 7.3 & 173 & 26.5 \\
\hline
\end{tabular}

Table 1. Residual strains after bed-in cycles, break loads, break strains and stiffness. 
When the twisted samples are tested to failure large differences in behavior are noted, Figure 4. Break loads and failure strains are also shown in Table 1. It should be emphasized that for all the tests to failure the initial gauge length was taken to be that measured at the end of bedding-in, so the bedding-in strain is not included in the results plotted in Figure 4 . As twist is introduced the response becomes progressively more non-linear. From a practical point of view, an indication of the change in stiffness is also of interest. A load range from 30 to $90 \mathrm{kN}$, i.e. 10 to $30 \%$ of the untwisted break load, was selected, which is a typical working load range offshore. A linear regression was taken using all the data points in that range. This value underlines the drop in apparent stiffness with twist.

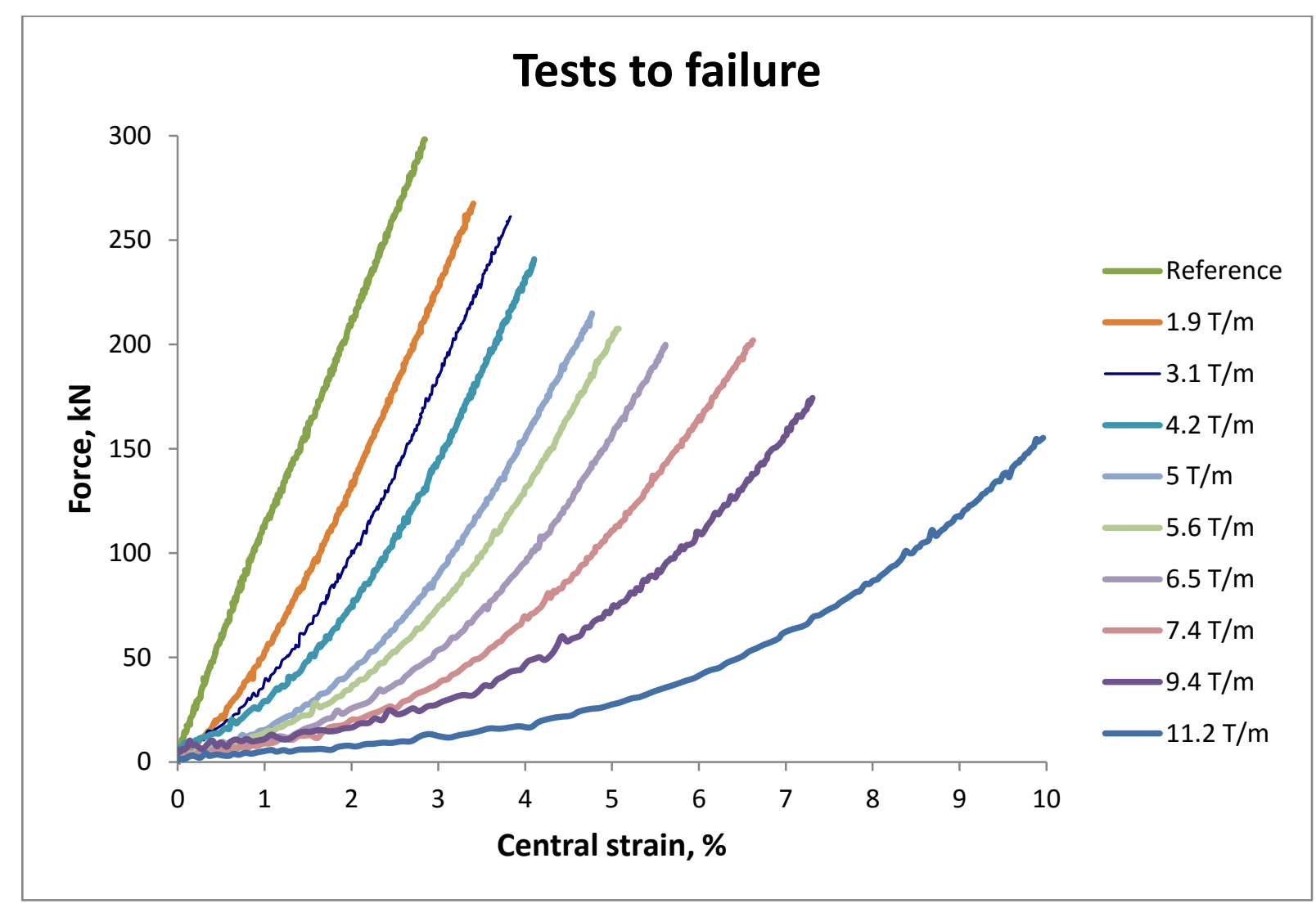

Figure 4. Force-central strain plots from tests to failure.

The failure strain increases significantly with twist, and the break loads fall in an almost linear way, Figure 5. 


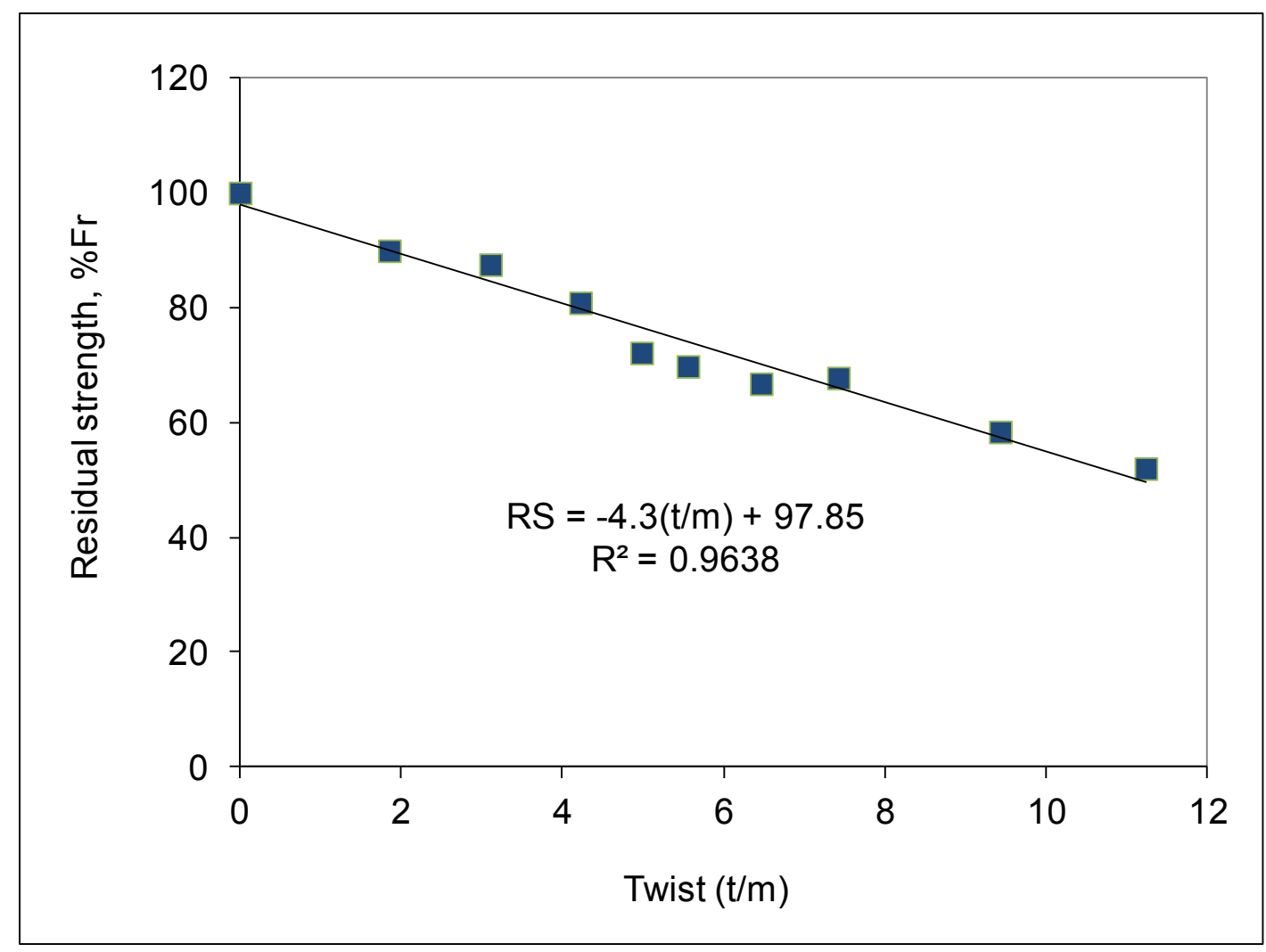

Figure 5. Residual strength (RS), as a percentage of untwisted value, versus twist.

\section{Model results.}

The numerical results were obtained from an in-house finite element code, dedicated to the modelling of the mechanical behaviour of entangled materials. This code, based on an implicit solver, determines the mechanical equilibrium of assemblies of beams of fibres, undergoing finite displacements and strains, and subjected to frictional contact interactions. The description of the basic models used in this approach to represent the behaviour of elementary beams and to detect and model frictional contact interactions can be found in [17].

This general simulation approach to the modelling of entangled fibrous materials is applied here to the case of synthetic braided ropes. Such structures result from the assembly of elementary fibres on three successive levels. At a first level, yarns are constituted from few thousand fibres. Strands are then formed by winding together few yarns (typically 5 to 8 yarns). Those yarns are eventually interlaced together by a braiding machine to form a rope. Considered ropes in this study are made of 12 strands, distributed in two crossing layers, and each yarn is made of 7 yarns. The rope is made of an assembly of 84 elementary yarns. 
Due to the limits regarding the number of components that can be handled in the FE code, the model is restricted to the scale of yarns and considers the rope as an assembly of 84 elementary yarns arranged into 12 strands. Each yarn is represented by a homogeneous beam, whose torsional stiffness and bending stiffness are adjusted so as to account for their high flexibility due to the relative motions allowed between filaments within the yarns.

The geometry of the initial configuration of the rope results from the braiding process and cannot be determined a priori. To face this difficulty, the proposed approach provides a way to determine this initial configuration: starting from an arbitrary configuration in which yarn trajectories are defined by double helices and interpenetrate each other, contact interactions are used to gradually separate penetrating yarns from each other, until getting a balanced configuration which satisfies contact conditions and fulfils the selected braiding pattern. This way of determining the initial configuration of the braided rope is explained in details in [16]. The obtained model was validated with a good agreement against experimental measures on a traction test, as reported in [16]. Only few parameters are required to define the model, namely: the axial stiffness of elementary yarns (determined experimentally), the radius of elementary yarns, the geometrical parameters for the arbitrary staring configurations (helix radii and helix pitches) and the braiding pattern.

The initial configuration was determined starting from a $680 \mathrm{~mm}$ long arbitrary configuration (Figure 6). 25200 beam elements and 50484 nodes where used to discretize the model. The main characteristics of the model are summarized in Table 2.

\begin{tabular}{|l|l|l|l|}
\hline Assembled yarn & Construction & Lay length & Friction coefficient \\
\hline $\begin{array}{l}\mathrm{E}=69291 \mathrm{MPa} \\
\text { Radius }=0.86 \mathrm{~mm}\end{array}$ & $\begin{array}{l}12 \times 7 \text { assembled } \\
\text { yarns }\end{array}$ & $136 \mathrm{~mm}$ & $\mathrm{Fr}=0.06$ \\
\hline
\end{tabular}

Table 2. Model input data 
Figure 6. Arbitrary starting configuration (top) and initial configuration of the braided rope determined by simulation (bottom)

Once the initial configuration has been determined, opposite incremental rotations about its axis were applied to the sample at both ends, until reaching different twists ranging from 1 $\mathrm{t} / \mathrm{m}$ to $8 \mathrm{t} / \mathrm{m}$. Afterwards an initial tensile load of $5 \mathrm{kN}$ was applied for each twist. Incremental axial displacements were then applied until failure. The considered failure criterion corresponds to a maximal axial stress of $2500 \mathrm{MPa}$. When the axial stress in a finite element exceeds this limit, the axial stiffness of this element is set to a value close to 0 . Simulations usually stopped due to divergence in the nonlinear algorithm after one or few yarns have broken.

Figure 7 shows the results from modeling of these tests, up to a twist of 5 turns/meter. For higher twist levels, simulations were less stable, and it was not possible to run them up to the breaking load. The model captures the change in shape of the load-strain plots as twist increases, with a falling break load and an increase in failure strain.

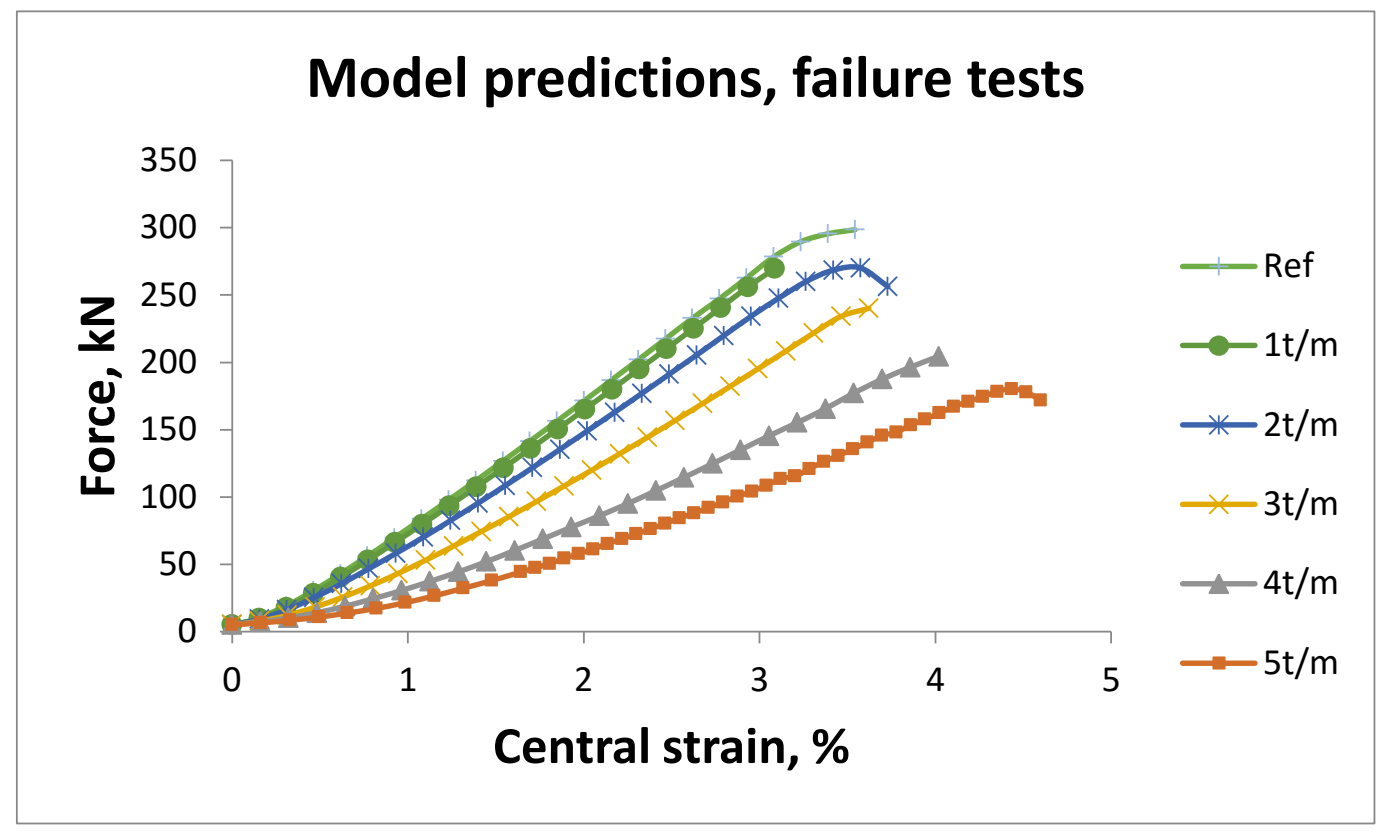

Figure 7. Model results for tensile tests on twisted ropes. 


\section{Test-Model Comparison and Discussion.}

Figure 8 shows the test-model comparisons for two cases, the reference sample, Figure 8a, and the 5 turns/meter case, Figure $8 \mathrm{~b}$.
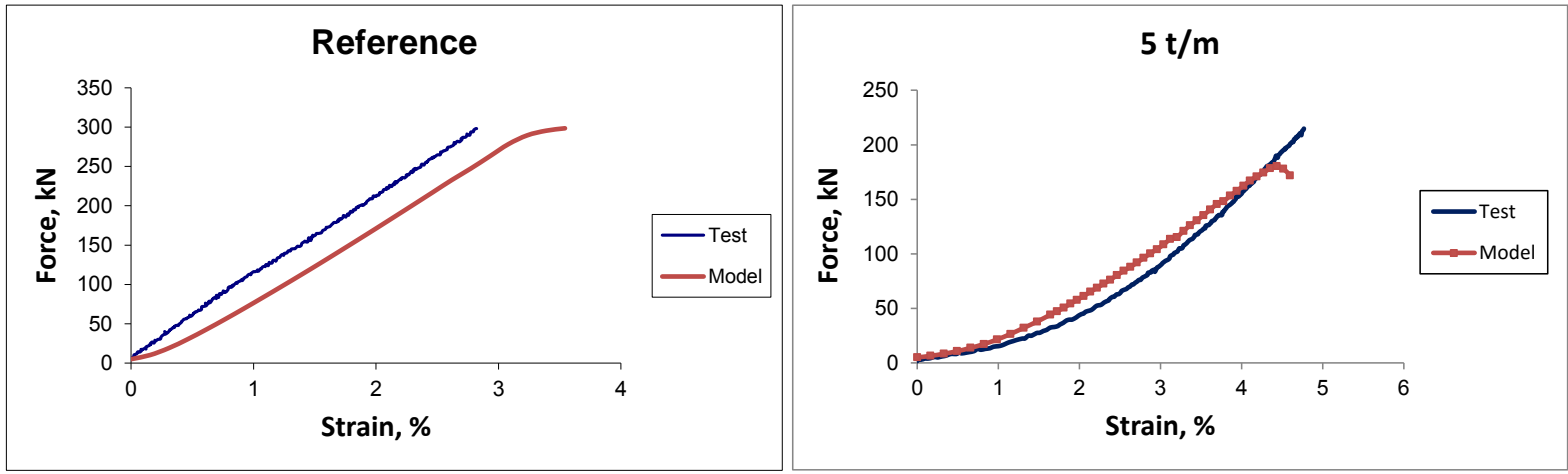

Figure 8. Test-model comparisons for a) reference sample (no twist), and b) sample with 5 turns/meter twist.

It should be emphasized that the model only uses measured assembled yarn data and rope geometry to predict the load-strain behavior of the rope, there is no adjustment, and the correlation with test data is quite good. In addition to providing the global response the model also allows individual elements to be inspected, the strands and their constituent yarns, in order to quantify changes in loads as the rope structure changes with twist. In addition to the mechanical response the model also provides unique information on the braid geometry and how it changes during testing. Figure 9 shows examples, which illustrate for each twist level the stresses in the braid at an applied tensile strain of $2.5 \%$. 


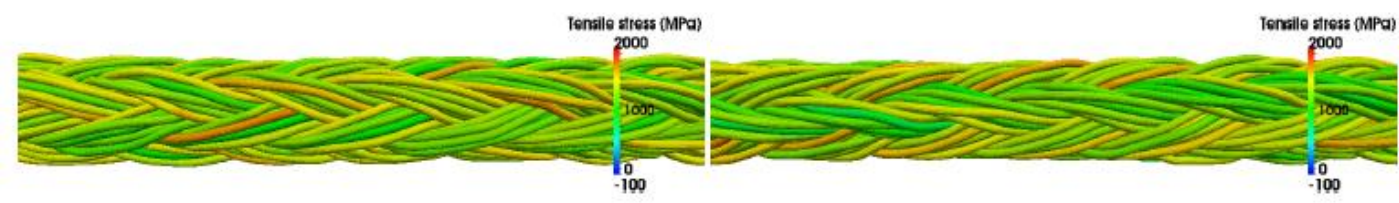

(a) Axial strain : $2.5 \%$ - Twist : $0 \mathrm{t} / \mathrm{m}$

(b) Axial strain : 2.5\% - Twist : $1 \mathrm{t} / \mathrm{m}$
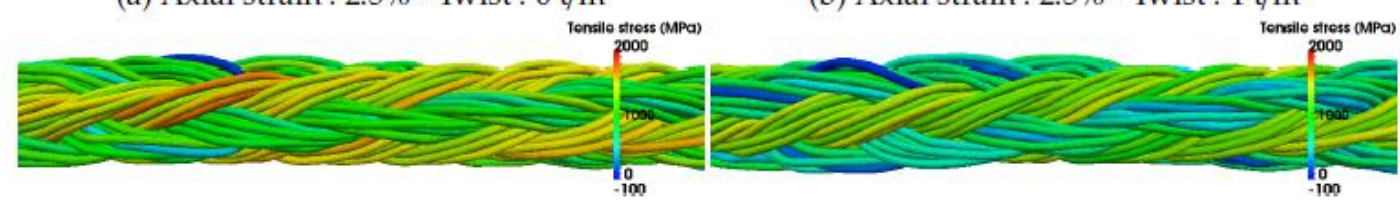

(c) Axial strain : $2.5 \%$ - Twist : $2 \mathrm{t} / \mathrm{m}$

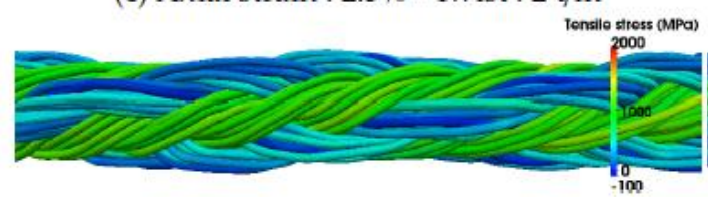

(d) Axial strain : $2.5 \%$ - Twist : $3 \mathrm{t} / \mathrm{m}$

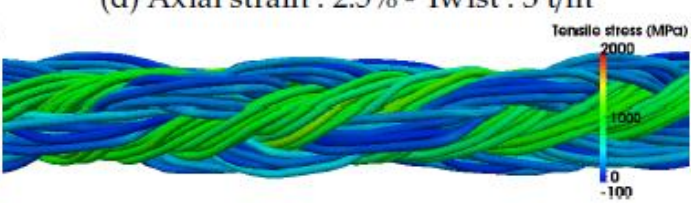

(e) Axial strain : $2.5 \%$ - Twist : $4 \mathrm{t} / \mathrm{m}$

(f) Axial strain : $2.5 \%$ - Twist : $5 \mathrm{t} / \mathrm{m}$

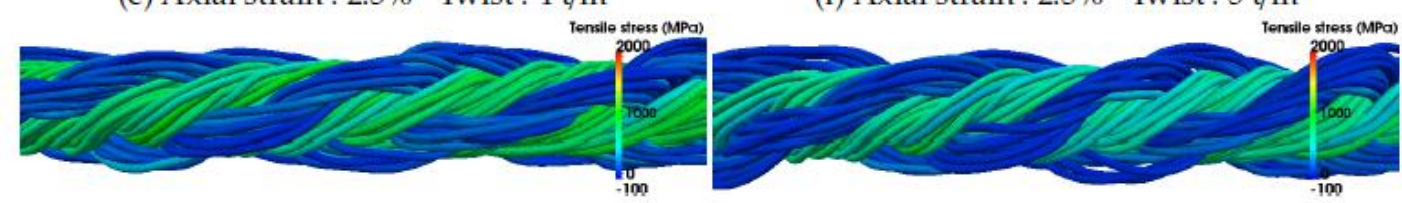

(g) Axial strain : $2.5 \%$ - Twist : $6 \mathrm{t} / \mathrm{m}$

(h) Axial strain : $2.5 \%$ - Twist : $7 \mathrm{t} / \mathrm{m}$

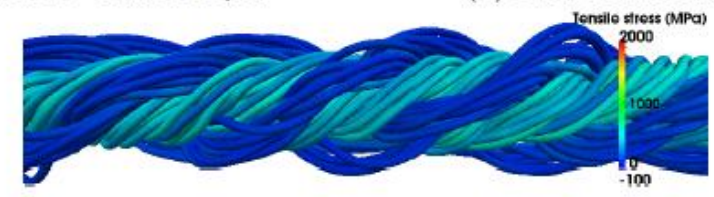

(i) Axial strain : $2.5 \%$ - Twist : $8 \mathrm{t} / \mathrm{m}$

Figure 9. Braid geometry and stress levels in assembled yarns during tensile tests, for an applied tensile strain of $2.5 \%$.

Physically, the following changes are introduced when a braid is twisted: First, the braid tends to shorten and the equilibrium between left hand and right hand strands is disturbed. When tensile loading is applied the overall rope length increases; depending on the twist level some or all of the imbalance between the strands may be restored, as the more highly loaded shorter lengths are extended and some redistribution of load will occur due to creep, resulting in longer strands progressively being loaded. Finally, when the rope is loaded to failure this process continues to high strain, with the amount of twist determining the proportion of strands which will be load-bearing when one of them reaches a critical load. When the twist level is sufficiently high to remove the contribution of all the strands in one 
direction (left hand or right hand depending on the twist direction) the load will drop to approximately $50 \%$ of the full untwisted break load. This distribution of the global axial load between left hand and right hand strands is illustrated in Figure 10, where axial stresses along all yarns are plotted for the $5 \mathrm{t} / \mathrm{m}$ case, almost at the end of the failure test; the average axial stress is almost three times higher in one layer than in the other one.

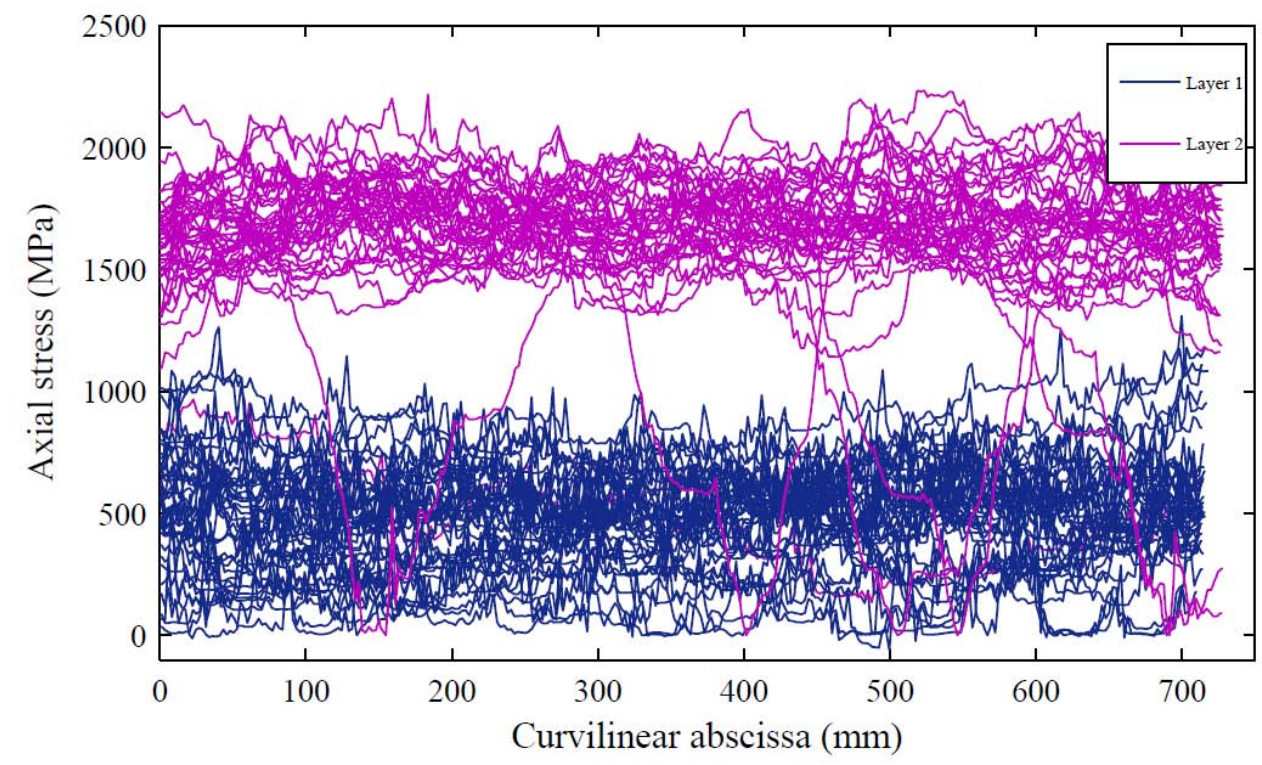

Figure 10. Distribution of axial stresses among all yarns from left hand strands (Layer 1) and right hand strands (Layer 2) for the sample twisted to $5 \mathrm{t} / \mathrm{m}$ just before failure (some of the yarns of Layer 2 are broken, which explains their local loss of axial stress)

The numerical model takes into account both the assembled yarn tensile behavior and the geometry. These are both measured. The only other input parameter is an internal friction value, taken to be 0.06 here. In order to examine the influence of this value, which is difficult to measure independently, a parametric study was performed in which the friction coefficient using two different coefficients of 0.06 and 0.10 . For the $5 \mathrm{t} / \mathrm{m}$ case Figure 11 shows that a significant increase of the friction coefficient only slightly increases the tensile load, but doesn't change its slope. 


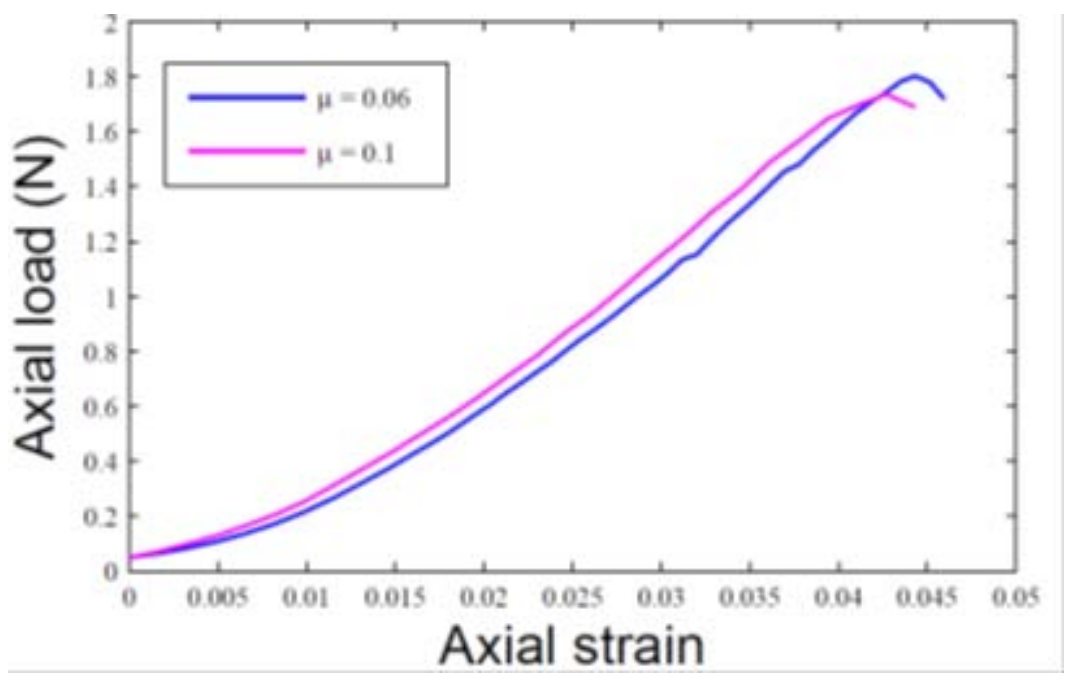

Figure 11. Influence of the friction coefficient: comparison of loading curves for the $5 \mathrm{t} / \mathrm{m}$ twist case, for friction coefficients of 0.06 and 0.10

Finally, it is interesting to compare the results from this data set to values measured previously for other rope sizes and geometries. Figure 12 shows a compilation of available HMPE rope data from a total of 43 tests, all normalized with respect to the nominal untwisted break loads. This plot should be used with caution, as some ropes were subjected to bedding-in cycles and different test machines were employed. While the rate of change of strength varies for different constructions and sizes there is a clear progressive drop in strength with increasing twist. A linear regression fit to all the data indicates a drop in residual strength of around $5 \%$ per turn per meter. The range of loss varies from $2 \%$ to $14 \%$ per turn per meter for these braided ropes, which cover a wide range of break loads, from 30 to 84 tons. This set of data should help to define an upper allowable twist limit for a required safety factor. 


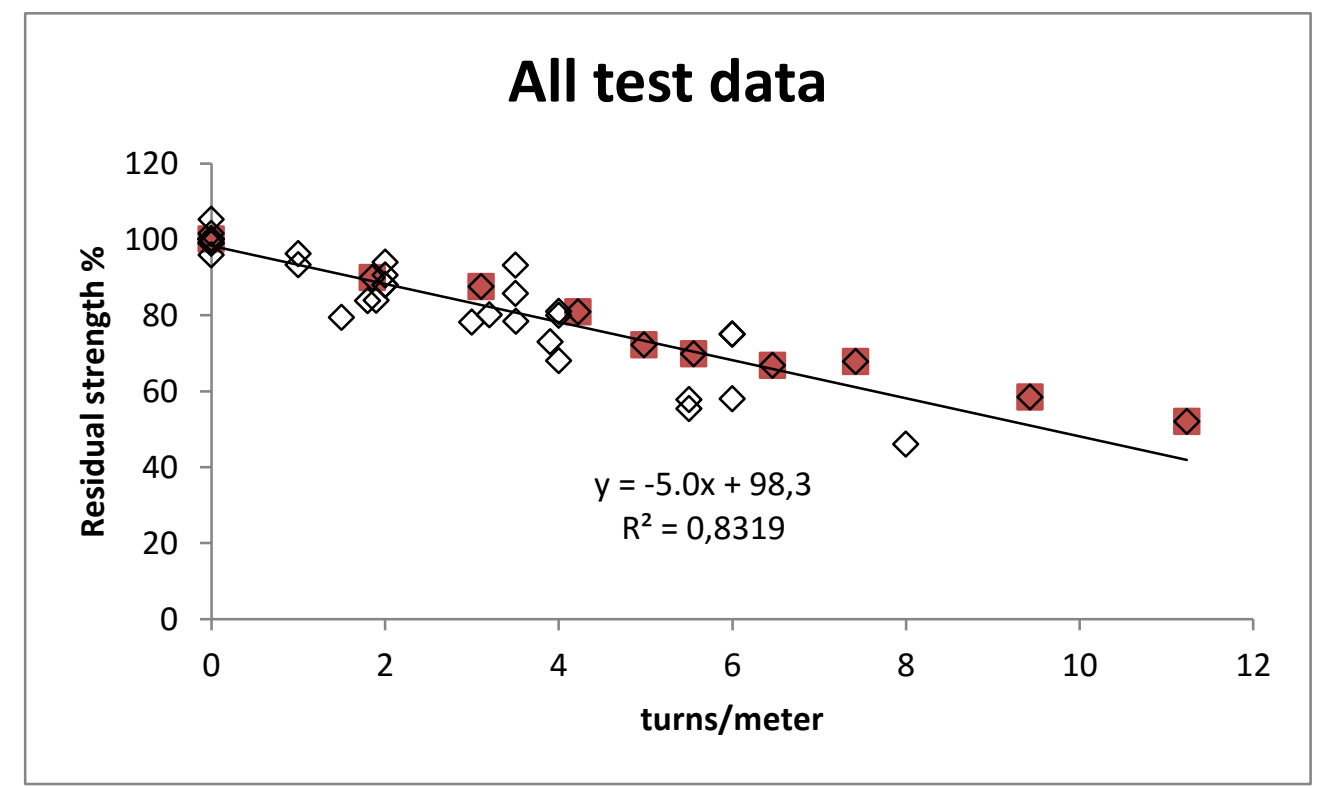

Figure 12. Compilation of current (red symbols) and published test data $[8,9]$ for 8 -strand and 12-strand braided HMPE ropes, with a linear fit to all data.

\section{Conclusions}

Braided ropes can be subjected to twisting in service. A new finite element model has been developed to simulate the effect of twist on both braid geometry and mechanical behavior under tensile loading of 12 -strand braided ropes. This provides a reasonable prediction of load-strain plots for a range of twist levels. Analysis of data from over 40 tests on 8- and 12strand braided ropes indicates a mean loss in residual strength of around 5\% per turn per meter. The development of the numerical constitutive model should now enable the parameters affecting twist sensitivity to be evaluated, in order to optimize fibre, finish and construction for applications where strength is critical.

\section{Acknowledgements}

The authors are grateful to Samson Ropes for the gift of materials for the experimental part of this study.

\section{References}

[1] McKenna HA, Hearle JWS, O'Hear N, Handbook of fibre rope technology, 2004, Woodhead Publishing. 
[2] Davies P, Reaud Y, Dussud L, Woerther P, Mechanical behaviour of HMPE and aramid fibre ropes for deep sea handling operations, Ocean Engineering, 38, 2011, pp. 2208-2214

[3] Smeets PJHM, Vlasblom MP, Weis JC, Latest improvements on HMPE rope design for steel wire rope applications OIPEEC 2009 / 3rd International Rope days - Stuttgart - March 2009

[4] Costello, GA (1997). Theory of wire rope. Springer Science \& Business Media.

[5] Chaplin CR, Torsional failure of a wire rope mooring line during installation in deep water, Engineering Failure Analysis , 6, 1998, 67-82.

[6] Ridge IMP, Tension-torsion fatigue behavior of wire ropes in offshore moorings, Ocean Engineering, 36, (2009), 650-660

[7] Chaplin, C. R., Rebel, G., \& Ridge, I. M. L. (2000). Tension/torsion interactions in multicomponent mooring lines, Proceedings Offshore Technology Conference, Paper OTC12173.

[8] Davies P, O'Hear N, Reduction in braided rope strength due to twist, Proc. OCEANS 07, Aberdeen, 2007.

[9] McCorkle E, Chou R, Stenvers D, Smeets P, Vlasblom M, Grootendorst E, Abrasion and residual strength of fibre tuglines, Proc ITS International Tug and Salvage convention, 2004.

[10] Leech CM, Banfield SJ, Overington MS, Lemoel M, The Prediction of Cyclic Load Behaviour and Modulus Modulation for Polyester and other Large Synthetic Fiber Ropes, Proceedings OCEANS 2003.

[11] Leech, CM, Hearle, JWS, Overington, MS and Banfield, SJ (1993). Modelling Tension and Torque Properties of Fibre Ropes and Splices, Proceedings Third International Offshore and Polar Engineering Conference (ISOPE), Singapore.

[12] Flory J, Leech CM, Banfield SJ, Pertruska D, Computer Model To Monitor Long-Term Performance and Integrity of Fiber Rope Mooring Lines, 2005 Offshore Technology Conference paper OTC-17592 
[13] Durville D., A Finite Element Approach of the Behaviour of Woven Materials at Microscopic Scale, in Mechanics of Microstructured Solids, J.-F. Ganghoffer, F. Pastrone (Ed.), Lecture Notes in Applied and Computational Mechanics, 46:39-46, 2009

[14] Durville D., Simulation of the mechanical behaviour of woven fabrics at the scale of fibers, International Journal of Material Forming, 3: 1241-1251, 2010

[15] Durville D., Microscopic approaches for understanding the mechanical behaviour of reinforcement in composites, in Composite reinforcements for optimum performance, $\mathrm{P}$. Boisse (Ed.), Woodhead Publishing Limited, 465-481, 2011

[16] Vu TD, Davies P, Durville D, Finite element simulation of the mechanical behaviour of synthetic braided ropes and validation on a tensile test, International Journal of Solids and Structures, Volume 58, April 2015, Pages 106-116

[17] Durville D., Contact-friction modeling within elastic beam assemblies: an application to knot tightening, Computational Mechanics, 49(6): 687-707, 2012. 